\title{
QUADRATURE OF INTEGRANDS WITH A LOGARITHMIC SINGULARITY
}

\author{
JOHN A. CROW
}

\begin{abstract}
A quadrature rule is presented that is exact for integrands of the form $\pi(\xi)+\varpi(\xi) \log \xi$ on the interval $(0,1)$, where $\pi$ and $\varpi$ are polynomials. The computed weights and abscissae are given for one- through seven-point rules. In particular, the four-point rule is exact for integral operators with logarithmically singular kernel on a cubic B-spline basis, and it is expected these results shall prove useful for numerical applications of weighted-residual finite element methods.
\end{abstract}

\section{INTRODUCTION}

In the formulation of solutions to initial value and boundary value problems for a partial differential equation by means of integral operators, one is led in a natural way to integrals with singular kernel functions. This is due to the singularity of the associated fundamental solution and the fact that this fundamental solution, or some derivative of it, often is used for the kernel of the integral representation. The problems arising in mathematical physics in two spatial dimensions typically lead to kernels with a logarithmic singularity. For example, the wave equation $\Psi_{t t}=c^{2} \Delta \Psi$ is reduced by the form $\Psi(t, \xi)=$ $\psi(\xi) e^{-i \omega t}$ to the Helmholtz equation $\Delta \psi+k^{2} \psi=0$, where $k^{2}=\omega^{2} / c^{2}$ and $\xi \in R^{2}$, and which in two dimensions has a fundamental solution involving $H_{0}^{(1)}(k|\xi|)$.

In numerical work based upon an integral representation with such a kernel, these integrals pose a bit of a problem for typically, as in a Galerkin method solution, many numerical integrals are required, but only particular ones (the so-called self-terms) possess a truly singular kernel and special handling of these terms must be performed. For the sake of concreteness, consider for the moment integrals of the form $\int_{0}^{1} f(\xi) d \xi$ where $f$ has a weak, integrable singularity at the lower endpoint. Two straightforward ways of computing such an integral are: (1) Replacing the lower endpoint by $\varepsilon(>0)$, and computing the resulting integrals for a sequence of $\varepsilon$ 's tending to zero, perhaps employing some sort of sequence extrapolation; and (2) decomposing $f$ into an easily-treated singular part $f_{s}$ of special form and a bounded part $f_{b}$ such that $f=f_{s}+f_{b}$, then computing the individual integrals by special techniques and

Received by the editor October 28, 1991.

1991 Mathematics Subject Classification. Primary 65D30, 65D32.

Key words and phrases. Numerical integration, quadrature formula, Galerkin method. 
TABLE 1

\begin{tabular}{|c|c|c|}
\hline N & Abscissae & Weights \\
\hline 0 & $0.367879441171442321595523770161(-0)$ & $0.100000000000000000000000000000(+1)$ \\
\hline 1 & 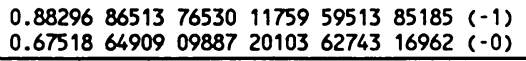 & $\begin{array}{llllllll}0.29849 & 98937 & 05524 & 91470 & 84741 & 43289 & (-0) \\
0.70150 & 01062 & 94475 & 08529 & 15258 & 56711 & (-0)\end{array}$ \\
\hline 2 & $\begin{array}{lllllll}0.28811 & 66253 & 09518 & 31174 & 32844 & 63059 & (-1) \\
0.30406 & 37296 & 12137 & 65261 & 08623 & 58639 & (-0) \\
0.81166 & 92253 & 44078 & 11686 & 37051 & 77761 & (-0)\end{array}$ & $\begin{array}{lllllll}0.10333 & 07079 & 64928 & 64676 & 92515 & 92664 & (-0) \\
0.45463 & 65259 & 70098 & 70884 & 06911 & 21425 & (-0) \\
0.44203 & 27660 & 64972 & 64439 & 00572 & 85911 & (-0)\end{array}$ \\
\hline 3 & 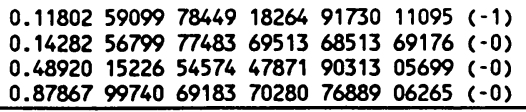 & 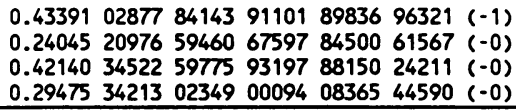 \\
\hline 4 & 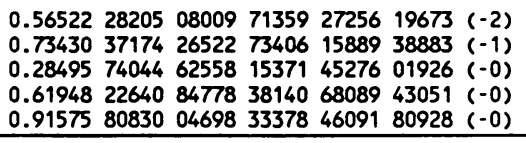 & 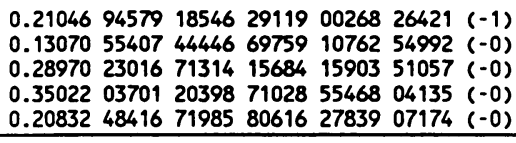 \\
\hline 5 & 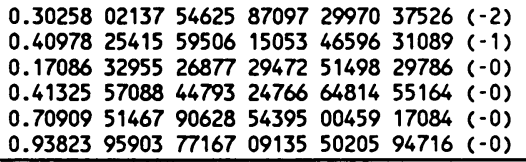 & $\begin{array}{lllllll}0.11351 & 33881 & 72726 & 09440 & 49112 & 38284 & (-1) \\
0.75241 & 06995 & 49165 & 22917 & 35628 & 91092 & (-1) \\
0.18879 & 00416 & 15416 & 35460 & 95079 & 43772 & (-0) \\
0.28582 & 07218 & 27227 & 31198 & 66834 & 80085 & (-0) \\
0.28448 & 64278 & 91408 & 80004 & 51516 & 69844 & (-0) \\
0.15431 & 03998 & 93758 & 40100 & 08094 & 93362 & (-0)\end{array}$ \\
\hline 6 & 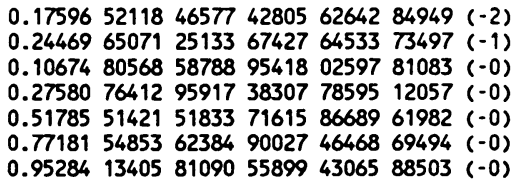 & $\begin{array}{llllllll}0.66326 & 66319 & 02570 & 51178 & 39049 & 89051 & (-2) \\
0.45799 & 70797 & 84753 & 34125 & 57673 & 48120 & (-1) \\
0.12384 & 02080 & 71318 & 19455 & 04895 & 64922 & (-0) \\
0.21210 & 19260 & 23811 & 93010 & 79148 & 75456 & (-0) \\
0.26139 & 06456 & 72007 & 72564 & 65806 & 06859 & (-0) \\
0.23163 & 61802 & 90909 & 38431 & 88155 & 26104 & (-0) \\
0.11859 & 86656 & 44451 & 72613 & 27836 & 41957 & (-0)\end{array}$ \\
\hline
\end{tabular}

summing the results. A simple example in which the latter approach might be applicable is seen in the evaluation of

$$
\int_{0}^{h} H_{0}^{(1)}(k \xi)\left[a+b \xi+c \xi^{2}\right] d \xi
$$

as the integrand naturally separates into a part which is well-behaved $\left(f_{b}\right)$ and another part which is a power series attached to a logarithm $\left(f_{s}\right)$. Other methods include change-of-variable techniques $[2,4,6,7,11]$ (the tanh, erf, and IMT rules), Gaussian quadrature for specialized singular weight functions [10], or most simply computing and ignoring the singularity. Evidently there are relative strengths and weaknesses associated with these approaches.

In this paper we describe a quadrature rule suited for integrands with and without logarithmic singularities. In particular, this rule

$$
\int_{0}^{1} f(\xi) d \xi=\sum_{n=0}^{N} w_{n} f\left(x_{n}\right)+\Re_{N+1}(f)
$$

is exact $\left(\mathfrak{R}_{N+1}(f)=0\right)$ for every $f$ of the form

$$
f(\xi)=\pi(\xi)+\varpi(\xi) \log \xi
$$

in which $\pi$ and $\varpi$ are polynomials of degree at most $N$. Thus, the interest lies in being able to compute numerically integrals, possibly possessing a logarithmic singularity in their integrands, without having to develop special methods for handling them or separating them from the nonsingular ones. The approach 
taken is similar in spirit to the one by Harris and Evans [5], appending singular basis functions to the usual (polynomial) quadrature basis. It is hoped that rules of this type will be found useful in computations in which a weakly singular integral operator is acting upon smooth functions as in the case when a spline basis is assumed in the Galerkin method. Low-order weights and abscissae $(N=0, \ldots, 6)$ are presented in Table 1. In applications of the finite element method, these low-order rules perhaps are the ones most useful.

\section{Derivation OF THE Weights AND ABSCISSAE}

Let $\mathfrak{P}_{N}$ be the linear space of polynomials on $[0,1]$ of degree at most $N$. We shall require a quadrature rule of the type (1) exact for every $f$ of the form (2) with $\pi, \varpi \in \mathfrak{P}_{N}$. Explicitly, we need

$$
\sum_{n=0}^{N} w_{n}\left[\pi\left(x_{n}\right)+\varpi\left(x_{n}\right) \log x_{n}\right]=\int_{0}^{1}[\pi(\xi)+\varpi(\xi) \log \xi] d \xi
$$

for every $\pi, \varpi \in \mathfrak{P}_{N}$. In particular, the choices $\varpi=0$ and $\pi=0$ lead alternately to the separate requirements

$$
\sum_{n=0}^{N} w_{n} \pi\left(x_{n}\right)=\int_{0}^{1} \pi(\xi) d \xi
$$

and

$$
\sum_{n=0}^{N} w_{n} \varpi\left(x_{n}\right) \log x_{n}=\int_{0}^{1} \varpi(\xi) \log \xi d \xi
$$

for each $\pi, \varpi \in \mathfrak{P}_{N}$. These must then hold for each basis function of $\mathfrak{P}_{N}$. A natural basis to select is from the set of power functions on $[0,1]$ and this choice leads to a Vandermonde system of equations; it is unfortunate though, that such systems are notorious for being ill-conditioned. When the numerical solution method to be described was used with this basis, condition numbers of this matrix varied quite a bit with estimates of $10^{8}-10^{15}$ being typical for the case $N=4$ prior to convergence. A more judicious choice is the shifted Legendre polynomials, $P_{\nu}^{*}(\xi)=P_{\nu}(2 \xi-1)$ [1]. There are two reasons for selecting these polynomials: First, they are orthogonal on $[0,1]$ and this ought to help in the conditioning of the problem; and second, all required integrals may be evaluated analytically and in a simple fashion. Then we must have for $\nu=0, \ldots, N$,

$$
\sum_{n=0}^{N} w_{n} P_{\nu}^{*}\left(x_{n}\right)=\int_{0}^{1} P_{\nu}^{*}(\xi) d \xi
$$

and

$$
\sum_{n=0}^{N} w_{n} P_{\nu}^{*}\left(x_{n}\right) \log x_{n}=\int_{0}^{1} P_{\nu}^{*}(\xi) \log \xi d \xi
$$

Upon evaluating the integrals (the final one is obtained from 2.17.8.2 of [8]) we obtain the following nonlinear system of $2 N+2$ equations in $2 N+2$ unknowns 
satisfied by the quadrature weights and abscissae:

$$
\begin{aligned}
\sum_{n=0}^{N} w_{n} P_{\nu}^{*}\left(x_{n}\right) & =\delta_{0, \nu}, \\
\sum_{n=0}^{N} w_{n} P_{\nu}^{*}\left(x_{n}\right) \log x_{n} & =g_{\nu},
\end{aligned}
$$

for every $\nu=0, \ldots, N$. Here, $g_{0}=-1$ and $g_{\nu}=(-1)^{1+\nu} / \nu(1+\nu)$ for $\nu=1, \ldots, N$. Numerical solution of this system, at least for the small $N$ of interest in applications of the finite element method, has been effected by iteration. For every $X=\left(X_{0}, \ldots, X_{N}\right) \in R^{N+1}$, let $A(X)$ be the linear operator associated with the matrix of elements $A_{i j}(X)=P_{i}^{*}\left(X_{j}\right)$. Then (3) and (4) are of the form

$$
A(x) w=d, \quad A(x) v(x, w)=g,
$$

where $v_{i}(x, w)=w_{i} \log x_{i}$ and $x$ and $w$ are the required abscissae and weights. Clearly, if we know the solution values of $w$ and $v$, the weights and abscissae are determined since $x_{i}=\exp \left(v_{i} / w_{i}\right)$. A one-step iteration is suggested naturally by the linearized system

$$
A(X) W=d, \quad A(X) v\left(X^{\prime}, W\right)=g,
$$

where here we regard $X$ as an estimate of the abscissae and $X^{\prime}$ a refinement of this estimate. We have $W=A(X)^{-1} d$ and $v\left(X^{\prime}, W\right)=A(X)^{-1} g$ so that the iteration is well defined for every invertible $A(X)$. In this way we have an iteration of the general form $X^{\prime}=\varphi(X)$.

\section{NUMERICAL RESULTS}

Clearly, if the iteration $X^{\prime}=\varphi(X)$ converges and the resulting $A(X)$ is nonsingular, then we have the desired quadrature rule; but it is unfortunate that in practice these iterates bounce about quite a bit- $\varphi$ is not a contraction. It was found though that the operator $A_{\mu}: R^{N+1} \rightarrow R^{N+1}$ with $A_{\mu} X=$ $X+\mu(\varphi(X)-X)$ tends to be contractive for $\mu \approx 1 / 2$. The resulting convergence is very slow (it appeared to be at most linear) but useful for providing starting values for a Newton-Raphson scheme for $F_{\mu}(x)=0$ with $F_{\mu}$ defined by $F_{\mu}(X)=\left(I-A_{\mu}\right) X$. A real problem for higher $N$ was obtaining reasonable estimates for the abscissae to begin the iteration, and the resulting convergence, or lack thereof, was rather sensitive to this initial choice; this sensitivity increases with increasing $N$ and suggests the need for using some sort of continuation method.

All computations were performed using about 50 significant decimal digits with the aid of Smith's FM multiple precision software [9]. The computed weights and abscissae to $30 \mathrm{~S}$ are given in Table 1 ; they have been checked and are believed to be accurate to all shown places.

\section{COMMENTS}

The set of integrands of the form (2) has the nice property that it is closed under scaling of the interval, for, if $f=\pi+\varpi \log$, then

$$
\begin{aligned}
f(h \xi) & =\pi(h \xi)+\varpi(h \xi) \log (h \xi) \\
& =(\pi(h \xi)+\varpi(h \xi) \log (h))+\varpi(h \xi) \log (\xi),
\end{aligned}
$$


which also is of the form (2). And since the quadrature rule presented evaluates $\int_{0}^{1} f(\xi) d \xi$ exactly, it also evaluates $\int_{0}^{h} f(\xi) d \xi$ exactly, which is a property not shared by most weighted Gaussian quadrature rules. This property makes the rule described attractive for applications in which weakly singular integrals must be evaluated over decreasing or partitioned meshes. It would be useful to establish the Kronrod-Patterson rules associated with the presented quadrature formulas.

\section{BIBLIOGRAPHY}

1. M. Abramowitz and I. A. Stegun (eds.), Handbook of mathematical functions, ninth printing, Dover, New York, 1965.

2. V. U. Aihie and G. A. Evans, Variable transformation methods and their use in general and singular quadratures, Internat. J. Comput. Math. 27 (1989), 91-101.

3. H. Engels and U. Eckhardt, Algorithm 33: Wilf-quadrature, Computing 18 (1977), 271-279.

4. G. A. Evans, R. C. Forbes, and J. Hyslop, The tanh transformation for singular integrals, Internat. J. Comput. Math. 5 (1984), 339-358.

5. C. G. Harris and W. A. B. Evans, Extension of numerical quadrature formulae to cater for end point singular behaviours over finite intervals, Internat. J. Comput. Math. (B) 6 (1977), 219-227.

6. M. Iri, S. Moriguti, and Y. Takasawa, On a certain quadrature formula, J. Comput. Math. 17 (1987), 3-20.

7. K. Murota and M. Iri, Parameter tuning and repeated application of the IMT-type transformation in numerical quadrature, Numer. Math. 38 (1982), 347-363.

8. A. P. Prudnikov, Yu. A. Brychkov, and O. I. Marichev, Integrals and series, vol. 2, Special functions, Gordon and Breach, New York, 1986.

9. D. M. Smith, Algorithm 693 : A FORTRAN package for floating-point multiple-precision arithmetic, ACM Trans. Math. Software 17 (1991), 273-283.

10. A. H. Stroud and D. Secrest, Gaussian quadrature formulas, Prentice-Hall, Englewood Cliffs, NJ, 1966.

11. H. Takahasi and M. Mori, Quadrature formulas obtained by variable transformations, Numer. Math. 21 (1973), 206-219.

12. H. S. Wilf, Exactness conditions in numerical quadrature, Numer. Math. 6 (1964), 315-319.

Autonetics Marine and Aircraft Systems Division, Rockwell International Corporation, 3370 Miraloma Avenue, Anaheim, CA 92803 